\title{
Direct Imaging of Oxygen Sub-lattice Deformation in Li-rich Cathode Material Using Electron Ptychography
}

Weixin Song ${ }^{1}$, Miguel Osorio ${ }^{2}$, John Marie ${ }^{3}$, Emanuela Liberti ${ }^{4}$, Xiaonan Luo ${ }^{1}$, Colum O'Leary ${ }^{5}$, Robert House $^{2}$, Peter Bruce ${ }^{2}$ and Peter Nellist ${ }^{6}$

${ }^{1}$ University of Oxford, Oxford, England, United Kingdom, ${ }^{2}$ University of Oxford, United States, ${ }^{3}$ University of OXFORD, United States, ${ }^{4}$ he Rosalind Franklin Institute, Harwell Campus, United Kingdom, ${ }^{5}$ University of California, Berkeley, United States, ${ }^{6}$ University of Oxford, Department of Materials, United States

Direct Imaging of Oxygen Sub-lattice Deformation in Li-rich Cathode Material Using Electron Ptychography

Weixin Song, Miguel P. Osorio, John J. Marie, EmanuelaLiberti, Xiaonan Luo, Colum O'Leary, Robert A. House, Peter G. Bruce, Peter D. Nellist

Department of Materials, University of Oxford, United Kingdom

Li-rich layered metal oxides, such as $\mathrm{Li}_{1.2} \mathrm{Ni}_{0.13} \mathrm{Mn}_{0.54} \mathrm{Co}_{0.13} \mathrm{O}_{2}$ offer much larger specific capacities ( $\left.>250 \mathrm{mAh} \mathrm{g}^{-1}\right)$ in Li-ion batteries than conventional oxides $\left(<200 \mathrm{mAh} \mathrm{g}^{-1}\right)$ because of the redox of lattice $\mathrm{O}^{2-}$ ions in addition to the cations. In the oxygen oxidation of $\mathrm{Li}_{1.2} \mathrm{Ni} 0.13 \mathrm{Mn}_{0 .}{ }_{4} \mathrm{Co}_{0.13} \mathrm{O}_{2}$, the lattice $\mathrm{O}^{2-}$ sublattice distorts in the bulk and surface region, with oxygen dimerization mainly occurring in the bulk and oxygen loss predominantly at the surface [1]. Observing the oxygen distortion is a key part to the understanding of oxygen redox [2]. Direct imaging of the oxygen deformation using atomic resolution electron microscopy is challenging because of the low contrast from oxygen and beam damage. Electron ptychography is a phase-reconstruction method in 4D scanning transmission electron microscopy (STEM), forming high-quality phase images because of its high signal-to-noise ratio and dose efficiency, and ability to detect and correct residual aberrations in post-acquisition data processing [3]. The ptychographic phase image is sensitive to both the heavy transition metal (TM) and the light $\mathrm{O}$ and Li atoms. Focused-probe electron ptychography allows simultaneous collection of aberration-corrected ADF image when recording the 4D datasets for phase retrieval [4]. Both the ADF and ptychographic phase images enable direct interpretation of the phase image and identification of the oxygen atoms.

Here, we show how to use focused-probe electron ptychography to investigate the average projected O-O distance of $\mathrm{Li} 1.2 \mathrm{Ni} 0.13 \mathrm{Mn} 0.54 \mathrm{Co} 0.13 \mathrm{O}_{2}$ at various stages in the first charge-discharge cycle and reveal the oxygen deformation in the lattice structure. Figures 1a and $\mathbf{b}$ display the simultaneous HAADF and ptychographic phase image of pristine $\mathrm{Li} 1.2 \mathrm{Ni} 0.13 \mathrm{Mn} 0.54 \mathrm{Co} 0.13 \mathrm{O}_{2}$ projected parallel the layers. Between the TM layers, the contrast from $\mathrm{O}$ and $\mathrm{Li}$ atoms is invisible in the HAADF but revealed in the phase image, enabling direct measurement of the projected distance illustrated in Figure1c. The statistical result of the atom distance is derived from multiple measurements using line profiles. Figure 1d shows the line profiles taken over the red lines. Such values from direct measurement can be distorted resulting from the sample drift during the slow-speed acquisition of the 4D datasets. To correct the values, we carried out multi-frame fast scanning of the HAADF images followed by rigid and non-rigid registration using SmartAlign [5] to generate zero-drift HAADF images and obtain the zero-drift TM-TM distances. Figures 2(a-c) display the zero-drift HAADF images of $\mathrm{Li} 1.2 \mathrm{Ni} 0.13 \mathrm{Mn} 0.54 \mathrm{Co} 0.13 \mathrm{O}_{2}$ at pristine, charged and discharged state, respectively. The zero-drift TM-TM distances can be used as a reference to calibrate the 
values directly measured from the ptychographic phase images of $\mathrm{Li} 1.2 \mathrm{Ni} 0.13 \mathrm{Mn} 0.54 \mathrm{Co} 0.13 \mathrm{O}_{2}$ at each charge-discharge stage. The measurement and calibration protocols generate zero-drift atom distances in $\mathrm{Li}_{1.2} \mathrm{Ni} 0.13 \mathrm{Mn} 0.54 \mathrm{Co} 0.13 \mathrm{O}_{2}$. This approach allows measurement of the changes in the layer spacing and distortion of the $\mathrm{O}$ octahedra coordinating the TM species during the first charge-discharge cycle [6].
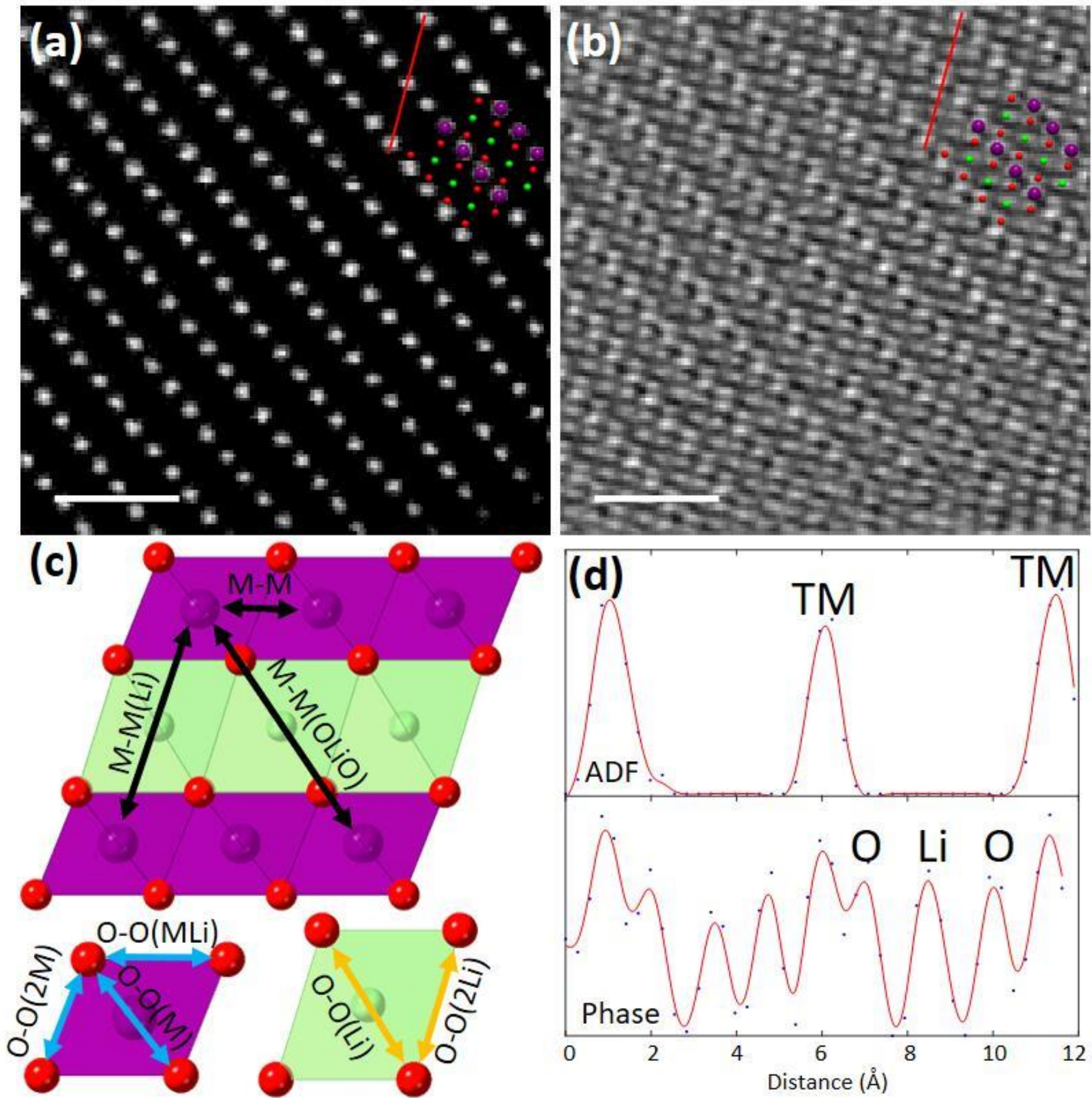

Figure 1. Figure 1 Measurement of projected atom distance in Li1.2[Ni0.13Mn0.54Co0.13]O2. Simultaneous (a) HAADF and (b) ptychographic phase image along [010] projection. Wigner distribution deconvolution algorithm is used for ptychographic reconstruction. Superposed is the crystal model, purple is TM, red $\mathrm{O}$ and green Li. Scale bar is $1 \mathrm{~nm}$. (c) Illustration of atom distance. In the named distances, the 
atoms in the brackets indicate where the bond projection crossing. (d) Line profiles across the red lines in the HAADF and phase image.

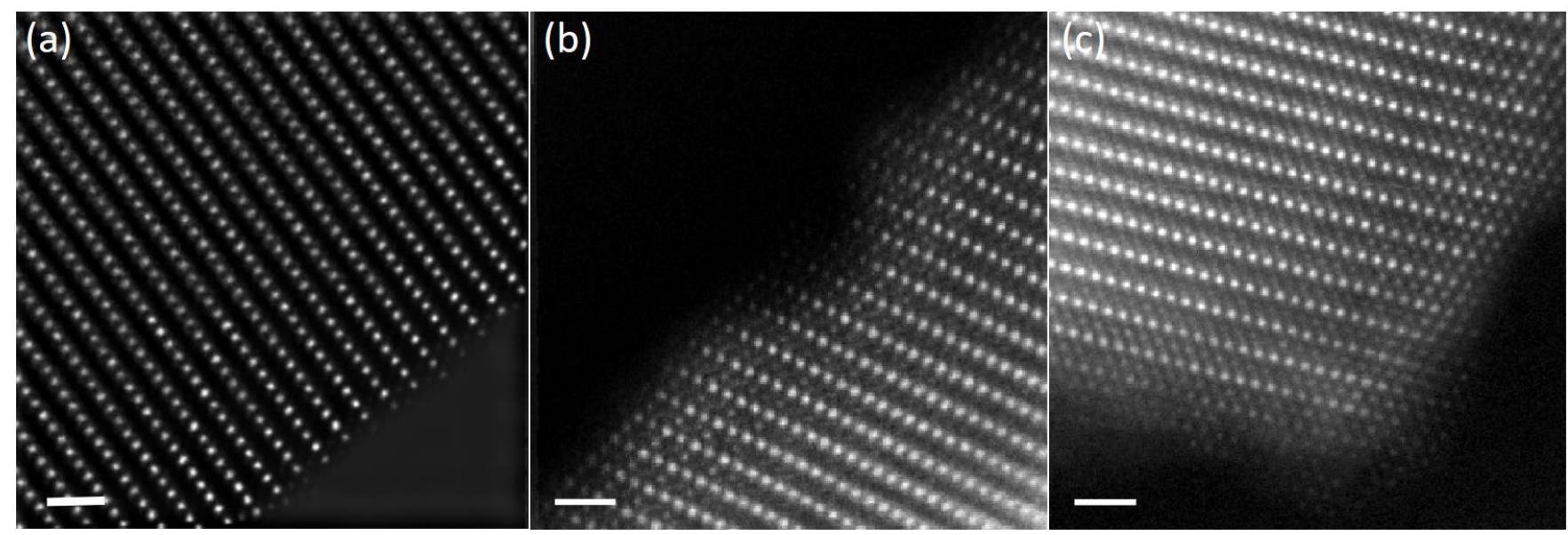

Figure 2. Figure 2 Measurement of reference distances from zero-drift HAADF image of Li1.2[Ni0.13Mn0.54Co0.13] 2 at (a) pristine sate, (b) charged state and (c) discharged state. The dose is 3720, 2050 and $1943 \mathrm{e}-/ \AA 2$, respectively. Scale bar is $1 \mathrm{~nm}$.

\section{References}

[1] House, R. A.; Rees, G. J.; Pérez-Osorio, M. A.; Marie, J.-J.; Boivin, E.; Robertson, A. W.; Nag, A.; Garcia-Fernandez, M.; Zhou, K.-J.; Bruce, P. G., Nature Energy 2020, 5 (10), 777-785.

[2] Liberti, E., et al., Ultramicroscopy 2019, 210, 112914.

[3] Yang, H.; MacLaren, I.; Jones, L.; Martinez, G. T.; Simson, M.; Huth, M.; Ryll, H.; Soltau, H.; Sagawa, R.; Kondo, Y.; Ophus, C.; Ercius, P.; Jin, L.; Kovács, A.; Nellist, P. D., Ultramicroscopy 2017, 180, 173-179.

[4] Lozano, J. G.; Martinez, G. T.; Jin, L.; Nellist, P. D.; Bruce, P. G., Nano Lett 2018, 18 (11), 68506855.

[5] Jones, L.; Yang, H.; Pennycook, T. J.; Marshall, M. S. J.; Van Aert, S.; Browning, N. D.; Castell, M. R.; Nellist, P. D., Advanced Structural and Chemical Imaging 2015, 1 (1), 8.

[6] The authors acknowledge use of characterization facilities within the David Cockayne Centre for Electron Microscopy, Department of Materials, University of Oxford and in particular the Faraday Institution (FIRG007, FIRG008), the EPSRC (EP/K040375/1 "South of England Analytical Electron Microscope") and additional instrument provision from the Henry Royce Institute (Grant reference EP/R010145/1). 